\title{
$\mathfrak{C b} \mathfrak{i} \mathfrak{i} \mathfrak{t} \mathfrak{l} \mathfrak{i} \mathfrak{d} \mathfrak{e} \mathfrak{s}$

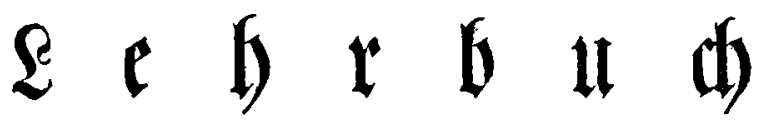

$\mathfrak{f} \mathfrak{u} \mathfrak{r}$

die $\mathfrak{I} \mathfrak{H} \mathfrak{g} \mathfrak{H}$ D.

$\mathfrak{B} \cup \mathbf{n}$

D. Sohann Georg Rofenmulles.

darl yottol ficsing inclabna. $181 \%$

$$
\text { Eiffte }\{\text { แ }
$$

$\{$ e $i \mathfrak{p}$ j $i$ g,

bey Beorg รดadim Gíd, 
